\title{
Introduction et présentation
}

Sous la direction de :

Michèle CLEMENT, Professeure de littérature française du XVI ${ }^{\mathrm{e}}$ siècle, Université Lumière Lyon 2, et directrice de l'École Doctorale Lettres, Langues, Linguistique et Arts, Lyon, France

\& Hervé MICOLET, Professeur de Littérature du XX ${ }^{\mathrm{e}}$ siècle, Université Lumière Lyon 2

AIR : c'est avec ce nom vaporeux que la Villa Gillet a mis en place depuis sept ans à Lyon un très solide parcours annuel de la production romanesque internationale. Séduite par l'ambition du projet, l'école doctorale «lettres, langues, linguistique et arts » l'a rejoint, en coulisses pourrait-on dire, depuis quatre ans. Des doctorants lisent une sélection de textes récemment parus, qui sont présentés aux Assises Internationales du Roman, travaillent pour écrire à plusieurs mains un article dans les trois mois qui précèdent les Assises, article qui est soumis à l'auteur puis discuté avec lui, en séance privée, en marge des Assises internationales. Les auteurs se sont chaque fois prêtés au jeu avec bonne grâce et même gourmandise, exposant le projet, la fabrique du livre et, bien au-delà, s'expliquant sur ce qui les nourrit. La littérature s'incarne alors, dans la voix, dans les silences, dans les corrections et les regrets : regret d'un premier livre pas comme on l'aurait voulu mais amour de ce livre qui fut le commencement (Jakuta Alikavazovic en 2013), confession cruellement indirecte sur la vieillesse «sans amour» des femmes (Pierre Pachet en 2012), avalanche de lectures en toute langue et de toute époque qui ouvre au monde (Alberto Manguel en 2011), chacun aborde sans rétention ses sujets de prédilection, ses parcours, ses butées et ses modèles et accepte d'en débattre dans un temps long (trois heures environ) avec un lectorat exigeant et curieux. Nous ne savons pas ce qu'en retirent les auteurs, mais il est sûr que les doctorants, au sortir de l'épreuve qui consiste à écrire sur un auteur qui lira ce que l'on a écrit de lui (expérience rarissime dans la recherche doctorale), ont une approche un peu différente de la pratique auctoriale, loin de celle représentée dans les émissions de télévision, dans les interview ultrarapides de la radio, ou sur les blogs internet à feuilleter et effeuiller. Le temps long de la lecture, de la mise en forme de sa lecture, de la confrontation avec celle d'autres lecteurs puis avec l'auteur, parfois interloqué, permet une immersion, une appropriation.

Si cette année - comme d'habitude - le choix a été mûrement pesé des trois livres retenus, pourtant au moment de cette sélection, dans l'hiver, nul n'aurait pu prévoir ce qui unirait si intimement ce petit corpus, en partie arbitraire. La Blonde et le bunker, 
L'Évaporation de l'oncle et Le Dépaysement. Voyages en France semblaient ouvrir des foyers d'interrogations très distincts, mais les doctorants après lecture, débat et écriture, en sont arrivés à saisir une proximité entre eux, au-delà des différences formelles, des projets esthétiques ou conceptuels très divergents : chacun des trois textes propose une enquête et se présente comme tel. La «teneur indicielle » des textes - pour reprendre une formule de JeanChristophe Bailly - est apparue à la lecture, et l'écriture des trois articles a été informée par ce principe de la recherche de traces, de signes, de liens au sein d'une réalité mouvante, en perpétuelle renégociation. C'est ce qui nous a paru pouvoir légitimer la réunion de ces trois articles sur trois objets aussi différents mais qui touchent le même point sensible.

Trois enquêtes, certes, mais aussi et surtout de véritables quêtes, et ce, avant tout pour les doctorants en charge de ce travail qui devient, au fil des séances, une expérience. Expérience de la communauté en premier lieu : celle d'échanges, de débats et de partages suite à une lecture des ouvrages sélectionnés, mais également d'autres œuvres des romanciers, ainsi que de l'apparat critique correspondant - lorsqu'il existe. Ainsi, des sensibilités, des points de vue divers et diversement informés se font face et se vivifient afin de tenter, au cours de longues tables rondes organisées en l'espace resserré d'un mois, de se nourrir et d'aboutir à une compréhension - aussi au sens originel, à un embrassement d'univers d'auteurs qui jusque-là demeuraient étrangers. C'est alors que l'expérience communautaire de l'écriture prend le relais, dans ses richesses, ses beautés et ses difficultés. Trois trios pour trois auteurs, et voilà que ces jeunes chercheurs habitués à travailler en solitaire doivent se confronter au penser et rédiger ensemble, à la réalisation d'un texte étymologiquement un tissage - commun, constitué d'opinions multiples, d'idées variées, mais aussi de voix : de styles et de manières propres à chacun. Le tout ayant pour ligne de mire l'unité. La difficulté du projet s'il en est réside dans cette quête de l'unité, sans unification ni homogénéisation, à l'image d'une mosaïque dont les fragments bariolés forment néanmoins un ensemble cohérent. Et soumettre, enfin. Soumettre les textes à la lecture et au regard critique des autres, des professeurs encadrants, mais aussi et surtout aux auteurs sur lesquels les doctorants ont travaillé.

L'intérêt de cette formation proposée par l'école doctorale est de pouvoir amener un autre point de vue sur le sacro-saint statut d'auteur à ces jeunes gens passionnés de littérature, sinon fascinés par elle. C'est en effet grâce, en second lieu, à l'expérience de dialogue, de conversation menée sur un pied d'égalité avec les écrivains choisis, que le projet trouve son 
point d'aboutissement. La solennité et la monumentalité presque magique que confère la seule prononciation du terme auteur se fendent alors pour laisser transparaître des humanités, des personnalités curieuses de savoir quel regard l'on peut porter sur leur travail et leur trajet - de carrière, mais aussi de vie -, et soucieuses également de connaître les êtres qui leur font face. L'on découvre une Christine Montalbetti à fleur de peau et de mots, à la conversation intime et libre, touchante dans sa pudeur et néanmoins avide de parler de ses œuvres, fragile dans son ouverture au regard et au sentiment de l'autre ; un Jean-Christophe Bailly franc, naturel et affirmé, dont l'engagement politique, esthétique ou éthique sans concession n'ont d'égales que sa prolixité et sa force de parole ; une Jakuta Alikavazovic enfin, au regard profond, sensible, abordable et pleine d'humour, désireuse de donner et transmettre, sous le voile de la modestie propre aux jeunes auteurs.

Un mois, c'est court et long à la fois. Court pour obtenir et ingérer les œuvres, ouvrages, articles nécessaires à la réalisation du projet - travail qui doit être effectué en surplus des recherches de doctorat ; mais long également, dans la véritable immersion au sein d'univers jusqu'alors inconnus, dans les étapes successives menées sous l'impératif de la rapidité. Long aussi parce que c'est un mois durant lequel les neuf participants furent hantés par leur rencontre avec les mots, et par la perspective de celle avec les êtres qui les ont assemblés. Un mois durant lequel ils sont passés de l'étrangeté à la convivialité d'un échange fondamental. Voilà de quoi alimenter ce qui est certainement la quête ultime de ces jeunes chercheurs : celle de la compréhension de ce rapport hors du commun qu'ils entretiennent avec la littérature et ses pourvoyeurs. 